\title{
Microfabrication of Anode Functional Layer in SOFC by 3D Printer
}

\author{
Kazuya TAKAHASHI $^{*}$, Hiroaki FuJITA ${ }^{2}$, Yuya ISHIKAWA ${ }^{2}$ and Takao NAKAGAKI ${ }^{2}$ \\ ${ }^{1}$ Graduate School of Environment and Energy Engineering, Waseda University, 1011 Honjo, Nishi-Tomida, Saitama 376-0035, \\ Japan \\ ${ }^{2}$ Department of Modern Mechanical Engineering, Waseda University, 3-4-1 Ookubo, Shinjuku-ku, Tokyo, 169-8555, Japan
}

\begin{abstract}
This work aims to increase the interface between anode and electrolyte in solid oxide fuel cells by controlling the 3D microstructure with a commercial ink-jet 3D printer. Anode and electrolyte inks suitable for use in a $3 \mathrm{D}$ printer were prepared by altering the viscosity and the droplet size. A porous anode structure that ensures a flow path for gases was achieved by addition of acrylic particles into the anode ink. A dense electrolyte structure that prevents leakage was created. The anode and electrolyte layers were produced as long, flat strips which were aligned in parallel to form sheets; these sheets were stacked orthogonally to complete the 3D microstructure called the 'anode functional layer'. The anode functional layer was roughly 100 micrometers on a side with a thickness of 4 micrometers. The anode functional layer was inserted between the anode and electrolyte. The assembled solid oxide fuel cell showed high performance when tested at $600{ }^{\circ} \mathrm{C}$ with dry methane as the fuel source.
\end{abstract}

\section{Introduction}

Solid oxide fuel cells (SOFC) are a highly efficient method of extracting electricity from hydrocarbon fuels which have received considerable interest as a next generation fuel cell. The electrochemical reaction of SOFCs occurs only at the triple-phase boundary (TPB); that is, the interface of the gas phase, the electron conduction phase, and the ion conducting phase. Though TPBs are distributed throughout the electrode, it has been reported that only TPBs located 10 to $20 \mu \mathrm{m}$ from the electrode-electrolyte interface contribute to the power generation reaction (Konno et al., 2010). Therefore, it is expected that increasing the density of TPB in the vicinity of the electrode-electrolyte interface will lead to a performance improvement. To these ends, Chen et al. (2008) proposed a method to increase the density of effective TPB sites by introducing a functional layer with a large quantity of TPB at the electrode-electrolyte interface (so-called, 'anode functional layer': AFL). Murayama et al. (2015) increased the depth of the effective TPB sites roughly 1.4 times, increasing the maximum power density of the SOFC by $\sim 2.2$ times.

This study investigates the potential to increase the thickness of the AFL in order to improve the performance of SOFCs. A commercially available inkjet-type 3D printer was used to fabricate the AFL by additive manufacturing. By controlling each phase constituting the $\mathrm{TPB}$, it was possible to generate a fine structure with increased effective TPB and improved performance. Prior to manufacturing, the $3 \mathrm{D}$-printed structure was modeled and the resultant performance was predicted via numerical calculation. Based on numerical results and limitations of the printer, a SOFC with finely-controlled $3 \mathrm{D}$ structure was fabricated and attested to determine the power generation performance.

\section{Performance Prediction by Numerical Calculation}

\subsection{Analysis object}

The performance improvement of SOFC depends not only on the TPB density, but also on the ion and electron transport resistance. As the transport resistance is fundamentally dependent on the geometry, alteration to the cell geometry was modeled and analyzed in COMSOL Multiphysics ${ }^{\circledR}$. The geometry of the microstructured cell is shown in Figure 1. Alternating strips of anodic and electrolytic material were printed directly atop the anode substrate, forming a microstructure sheet. After printing a single microstructured sheet, the anode substrate was rotated 90 degree and another layer of anode and electrolyte strips were printed atop the previous microstructured sheet. This build-up of microstructured sheets forms the AFL. The strip width, strip height, and number of microstructured sheets were varied to evaluate the impact on overvoltage. Considering the precision of the inkjet type 3D printer with $21.5 \mu \mathrm{m}$ nozzles, strip width was set to 50,100 , and $150 \mu \mathrm{m}$. Strip height was varied from 1 to $5 \mu \mathrm{m}$ in $1 \mu \mathrm{m}$ steps. The number of layers 
of microstructured sheets was set to 2 and 4 . For comparative purposes, a conventional SOFC (i.e., lacking a designed AFL) was simulated by one-dimensional analysis.

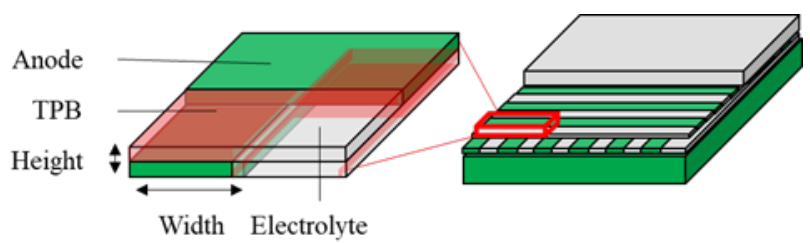

Figure 1. Schematic view of the microstructure

\subsection{Numerical model}

The structure described in Section 2.1 was built on COMSOL modeler as shown in Figure 2 with the structure of periodic boundary along the $x$ and $y$ axes. In Figure 2, the width and height of strips are given by $w_{a}$ and $h_{a}$, respectively.

The temperature and pressure were held constant at $1000^{\circ} \mathrm{C}$ and $1.013 \times 10^{5} \mathrm{~Pa}$. The inflowing fuel gas was hydrogen and water vapor, and the hydrogen gas partial pressure was held constant at $0.993 \times 10^{5} \mathrm{~Pa}$ on the current collecting surface. The current density on the current collecting surface and electrolyte interface were equal and set constant. The degree of tortuosity (i.e., void space, Ni, Yttria stabilized zirconia: YSZ) through the anode was based on measurements by focused ion beam scanning electron microscopy (Shikazono et al., 2009).

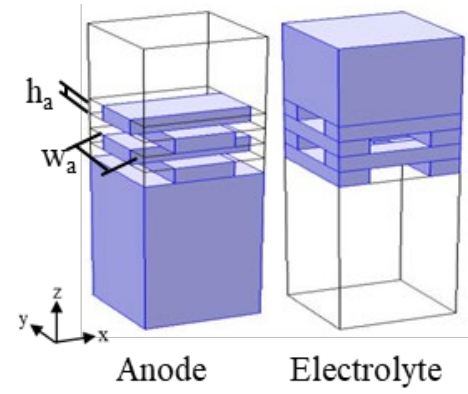

Figure 2. 3D model of numerical calculation

\subsection{Governing equations}

The governing equations applied to the void, electrode layer, and electrolyte layer were the gas, electron, and ion diffusion equations, respectively (Eqs. (1), (2) and (3)). Using the Stefan-Maxwell model (Eq. (4)) for fuel gas diffusion, combined with Graham's law (Eq. (5)), the diffusion coefficient was calculated per Eq. (6) and Eq. (7). In order to carry out numerical calculation for overvoltage reduction by microstructure, the hydrogen was selected instead of methane with overvoltage due to carbon deposition. The various effective diffusion coefficients are defined by Eq. (8). The reaction current at the electrolyte-anode interface, $i_{c t}$ in Eqs. (1), (2), and (3) is given by Eq. (9) using the Butler-Volmer type equation (Chan and Xia, 2001). Local activation overvoltage was defined as the difference of the local equilibrium in the oxide from the electrode potential of the equilibrium state and expressed in Eq. (10) (Mizusaki et al., 1987). In addition, the overpotential of the whole anode $\left(\eta_{\text {anode }}\right)$ is expressed by Eq. (11). The exchange current density $\left(i_{0}\right)$ was calculated from the empirical formula of de Bore (1998) as shown in Eq. (12).

$$
\begin{aligned}
& 2 F \nabla \cdot\left(D \nabla C_{H_{2}}\right)=i_{c t} \\
& \nabla \cdot\left(\frac{\sigma_{e^{-}}}{F} \nabla \mu_{e^{-}}\right)=-i_{c t} \\
& \nabla \cdot\left(\frac{\sigma_{o^{2-}}}{2 F} \nabla \mu_{o^{2-}}\right)=i_{c t} \\
& \frac{N_{i}}{D_{i, k}}+\sum_{j=1, j \neq i}^{n} \frac{y_{j} N_{i}-y_{i} N_{j}}{D_{i, k}}=-\frac{1}{R T} \nabla p_{i} \\
& \sum_{i=1}^{n} N_{i} \sqrt{M_{i}}=0 \\
& D=\left[\frac{1-\alpha y_{H_{2}}}{D_{H_{2}, H_{2} O}}+\frac{1}{D_{H_{2}, k}}\right]^{-1} \\
& \alpha=1-\sqrt{\frac{M_{H_{2}}}{M_{H_{2} O}}} \\
& D_{i, e f f}=\frac{\varepsilon_{i}}{\tau_{i}} D_{i} \\
& i_{c t}=i_{0} L_{T P B}\left\{\exp \left(\frac{2 F}{R T} \eta_{a c t}\right)\right. \\
& \left.-\exp \left(-\frac{F}{R T} \eta_{a c t}\right)\right\} \\
& \eta_{a c t}=-\frac{1}{2 F}\left\{2 \mu_{e}-\mu_{o^{2-}}\right. \\
& \left.+\left(\Delta G+R T \log \frac{P_{\mathrm{H}_{2} \mathrm{O}}}{P_{\mathrm{H}_{2}}}\right)\right\} \\
& \eta_{\text {anode }}=-\frac{1}{2 F}\left\{2 \mu_{e^{-}, \text {bulk }}-\mu_{O^{2-}, \text { lyte }}+\Delta G\right. \\
& \left.+R T \log \left(\frac{P_{\mathrm{H}_{2} \mathrm{O}, \text { bulk }}}{P_{\mathrm{H}_{2}, \text { bulk }}}\right)\right\} \\
& i_{0}=31.4 P_{H_{2}}^{-0.03} P_{H_{2} \mathrm{O}}^{0.4} \exp \left(-\frac{1.59 \times 10^{5}}{R T}\right)
\end{aligned}
$$

\subsection{Numerical calculation result}

Figure 3 shows the effect of the number of sheets on overvoltage compared to no AFL when the strip height $h_{a}$ is $2 \mu \mathrm{m}$. The overvoltage in this calculation model is that from AFL-electrolyte interface to the anode current collecting surface. It was confirmed that the overvoltage decreased as the number of layers of microstructured sheets increased. The increase in layers of microstructured sheets naturally adds electrolyte-anode interfaces, and thus TPB sites. It is postulated that the increase in TPB 
sites is the source of overvoltage reduction. These simulation results suggest that control of the microstructure can improve performance.

In Figure 4, it is shown that while both $w_{a}$ and $h_{a}$ effect the overvoltage, the influence of $h_{a}$ is less pronounced than that of $w_{a}$. The large $w_{a}$ relative to $h_{a}$ of this microstructure means the influence of $h_{a}$ on the overvoltage is minor, because the reaction rate is controlled by the conduction of oxide ion. Additionally, the reduction of $w_{a}$ is believed to reduce the overvoltage by decreasing the transport distance of oxide ions. These results confirmed that increasing the number of layers of structured sheets and decreasing $w_{a}$ should reduce the overvoltage.

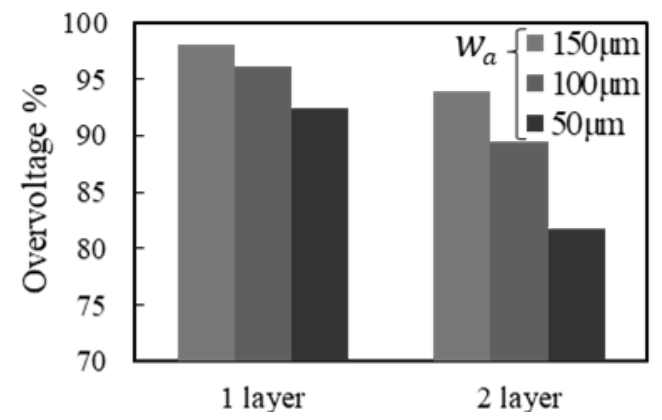

Figure 3. Effect of number of layers on overvoltage compared to no AFL

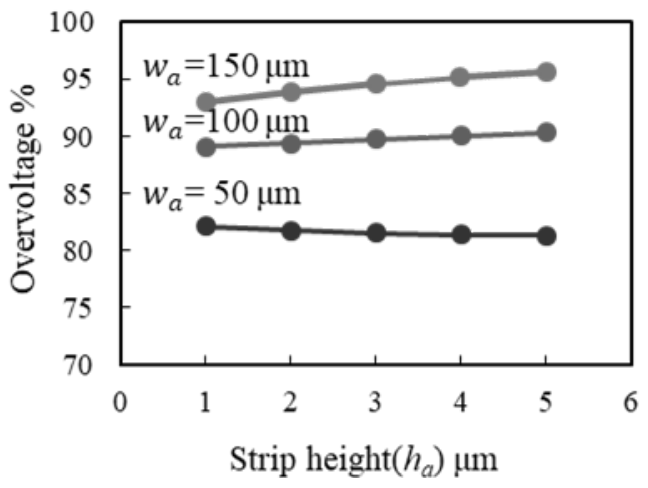

Figure 4. Overvoltage vs. strip width and height (No $\mathrm{AFL}=100 \%$ )

\section{3D Printing and Cell Testing}

\subsection{Material inks preparation}

Two types of ink were used to fabricate the microstructure using the "Dimatix", a inject printer produced by Fujifilm Corporation: one for the electrolyte and one for the anode. The composition ratio of each ink is shown in Table 1. The solute was adjusted to 6.1 volume percent of the solution. Acrylic particles with a diameter of $0.8 \mu \mathrm{m}$ were added to the anode ink; these particles are volatized during the sintering process described in Section 3.3, forming the void spaces through which fuel gas passes. Cracks in the electrolyte strongly diminish SOFC performance. To suppress cracking during cell sintering, polyvinyl butyral (PVB) was added to polyvinyl pyrrolidone (PVP), which is used as a binder in the electrolyte ink. The increase in viscosity due to PVB addition was counteracted by the addition of $\mathrm{N}$ methylpyrrolidone (NMP), which is a low viscosity organic solvent, because viscosity is limited due to clogging the inkjet nozzles.

Table 1. Ink compositions used in the 3D printer

\begin{tabular}{ccc}
\hline & Electrolyte & Anode \\
\hline Solvent & NMP & Benzyl alcohol \\
\hline Solute & $\begin{array}{c}\text { Gadolinium-doped Cerium } \\
\text { (GDC) }\end{array}$ & $\begin{array}{c}\text { NiO } \\
\text { acrylic }\end{array}$ \\
\hline \multirow{2}{*}{ Binder } & $\begin{array}{c}\text { PVP } \\
\text { PVB }\end{array}$ & PVP \\
\hline
\end{tabular}

\subsection{Printed 3D microstructure}

The structure theoretically analyzed in Section 2 was fabricated by 3D printing. Firstly, single microstructured sheet was formed as shown in Figure 1 and thereafter the anode substrate was rotated 90 degrees. The anode strips were printed and electrolyte strips were formed between the anode strips atop the single microstructured by way of dip-coating. The structure generated by $3 \mathrm{D}$ printing is shown schematically in Figure 5. The strip width of this microstructure was $100 \mu \mathrm{m}$, and the strip height was $2 \mu \mathrm{m}$.

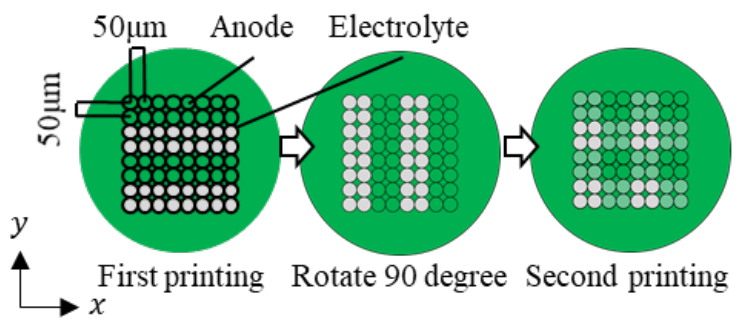

Figure 5. Image of the microstructure printing process

\subsection{Cell preparation}

The anode substrate was formed from the equivalent components as the anode ink. After mixing the NiO, GDC, and acrylic particles in ethanol for 48 hours in a ball mill, the anode substrate was dry press-molded and sintered at $900^{\circ} \mathrm{C}$ for 2 hours in air. This sintering volatized the acrylic particles. The AFL was then printed atop the anode substrate as described in Section 3.2. After printing, a thin electrolyte film was formed atop the AFL by dip coating. The entire cell was sintered at $1350{ }^{\circ} \mathrm{C}$. The cathode material was fabricated in the same manner as the anode substrate using the composition in Table 2. The cathode was applied atop the electrolyte by hand and the entire cell was sintered at $1110^{\circ} \mathrm{C}$ for 2 hours.

Table 2. Cathode compositions

\begin{tabular}{cc}
\hline & Cathode \\
\hline \multirow{2}{*}{ Solvent } & Ethanol \\
& $\alpha$-terpineol \\
\hline \multirow{2}{*}{ Solute } & LSCF \\
& GDC \\
\hline
\end{tabular}




\begin{tabular}{cc}
\hline Binder & PVP \\
\hline
\end{tabular}

\subsection{Power generation test}

A power generation test was carried out under the following test conditions using the equipment set-up shown in Figure 6. Mixture gas of nitrogen and methane and compressed air are supplied into anode and cathode side, respectively. To prevent diffusion resistance in the boundary layer and porous electrodes, both gas utilization factor was set to $<5 \%$ for individual total flow rate. Prior to power generation tests, the temperature was increased to $600^{\circ} \mathrm{C}$ under a hydrogen atmosphere in anode side for one hour to reduce nickel oxide to Ni. The fabricated anode-supported cells were evaluated by power density and i-v characteristic under three temperatures: 500, 550, and $600^{\circ} \mathrm{C}$.

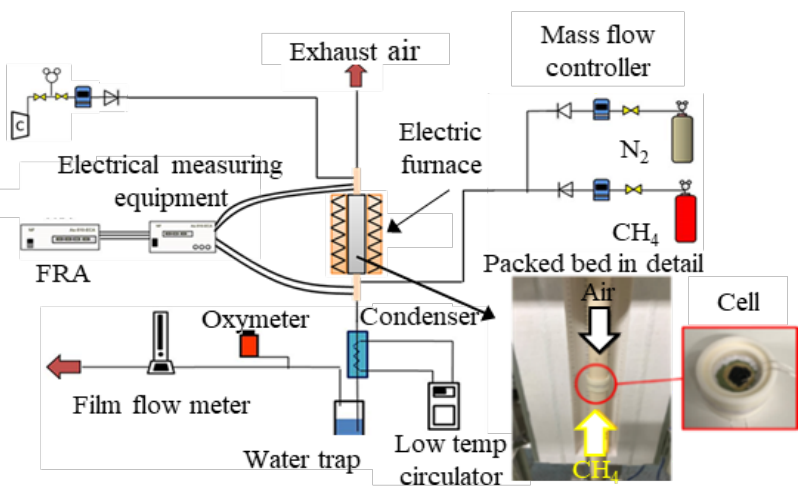

Figure 6. Power generation test equipment

\subsection{Structure observation and performance evaluation}

The microstructure of two orthogonally printed structured sheets was observed by scanning electron microscopy (SEM), and it was confirmed that no cracks were present (Figure 7) with properly fabricated microstructure. Laser microscopy was used to measure the microstructured sheet height. All microstructured sheet layers were found to be roughly $2 \mu \mathrm{m}$, equivalent to that assumed in the numerical calculation. According to the power generation test results, under dry methane conditions, the maximum power density was $122 \mathrm{~mW} / \mathrm{cm}^{2}$ at $500^{\circ} \mathrm{C}, 197 \mathrm{~mW} / \mathrm{cm}^{2}$ at $550^{\circ} \mathrm{C}$, and $302 \mathrm{~mW} / \mathrm{cm}^{2}$ at $600^{\circ} \mathrm{C}$. The results are aggregated in Figure 8. Figure 9 compares the results of a cell with (solid lines) and without (dashed lines) a controlled 3D microstructure at $600^{\circ} \mathrm{C}$. As shown in Figure 9, the controlled 3D microstructure increased the maximum output power density slightly, but it was poorer than expected. To investigate the cause of this unexpected result, the sources of overvoltage were disaggregated into the activation overvoltage, resistance overvoltage, and concentration overvoltage as shown in Figure 10(a), 10(b), and $10(\mathrm{c})$, respectively. Activation overvoltage and resistance overvoltage clearly decreased with the inclusion of a 3D microstructure. On the other hand, the 3D microstructure increased the concentration overvoltage at current densities above $500 \mathrm{~A} / \mathrm{cm}^{2}$. As the countermeasure, increasing the porosity in the anode substrate is likely to improve overall performance.

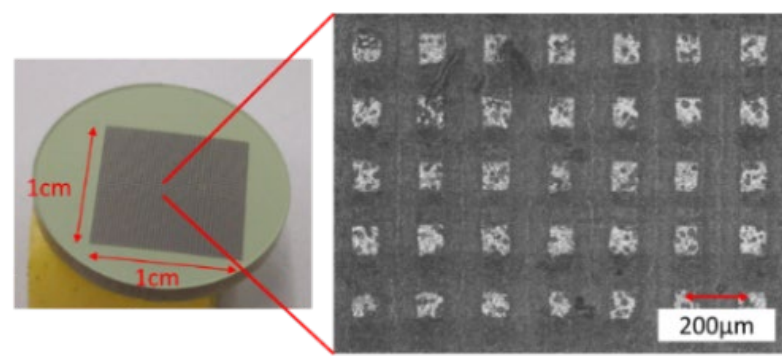

Figure 7. SEM image of the AFL microstructure

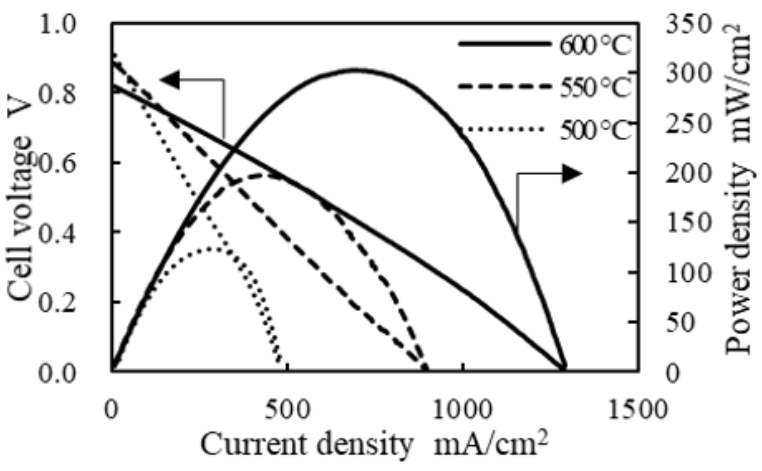

Figure 8. 3-D fabricated SOFC empirical performance

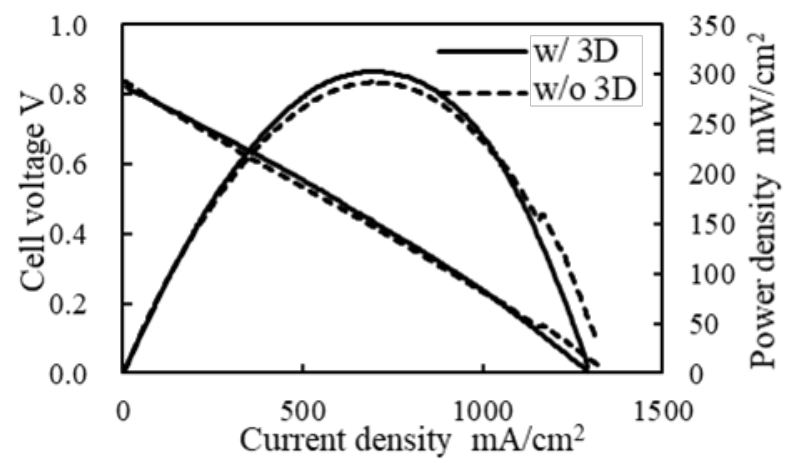

Figure 9. Comparison with and without microstructure

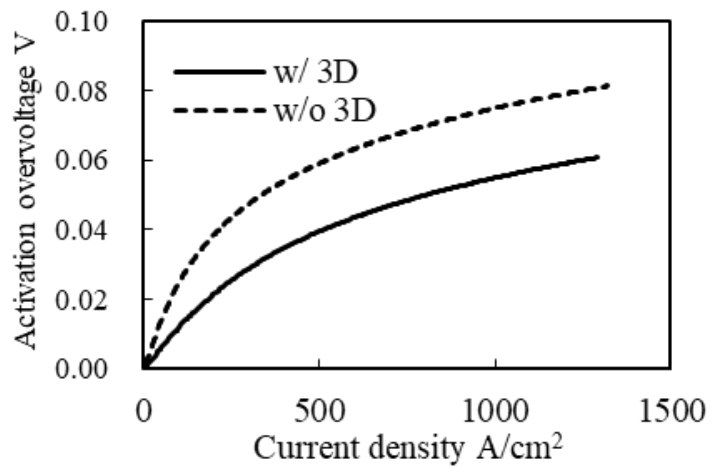

Figure 10(a). Comparison of activation overvoltage 




Figure 10(b). Comparison of resistance overvoltage

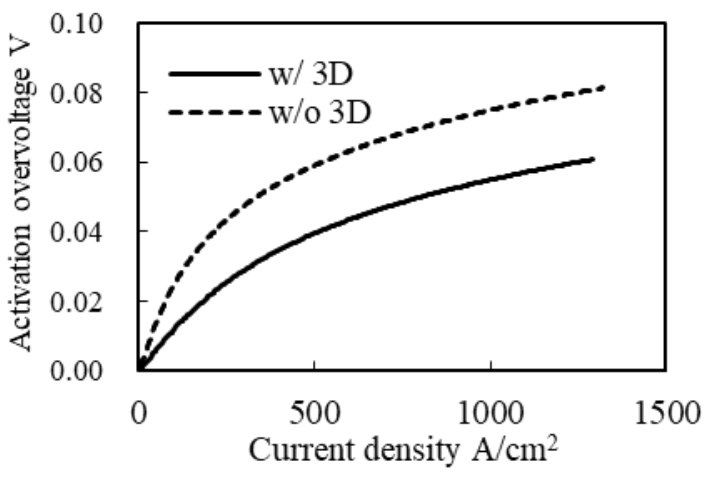

Figure 10(c). Comparison of concentration overvoltage

\section{Conclusion}

In this study, the triple phase boundary density at the anode-electrolyte interface of a solid oxide fuel cell was enlarged by controlled fabrication of the microstructure using an inkjet-type 3D printer.

The reduction in overvoltage of cells with an engineered microstructure was confirmed. Simulation results indicate that the mechanism reducing overvoltage is the decrease in the strip width and the increase in the number of layers of structured sheets. The minimum strip width and height that can be produced using the current equipment and inks was determined to be 50 and $2 \mu \mathrm{m}$, respectively.

The 3-D fabricated SOFC generated a maximum power density of $302 \mathrm{~mW} / \mathrm{cm}^{2}$ under dry fuel conditions at 600 ${ }^{\circ} \mathrm{C}$. This power density was equivalent to a SOFC without a controlled 3D microstructure. This unexpected result was posited to be due to concentration overvoltage, which could be rectified by increasing porosity.

\section{Symbol}

$D_{i} \quad$ Diffusion coefficient $\left[\mathrm{m}^{2} / \mathrm{s}\right]$

$D_{e f f, i} \quad$ Effective diffusion coefficient of species $i\left[\mathrm{~m}^{2} / \mathrm{s}\right]$

$F \quad$ Faraday constant $[\mathrm{A} / \mathrm{mol}]$

$\Delta G \quad$ Gibbs free energy-change [J/mol]

$i_{c t} \quad$ Generation term at TPB interface $\left[\mathrm{A} / \mathrm{m}^{2}\right]$

$i_{0} \quad$ Exchange current density per TPB $\left[\mathrm{A} / \mathrm{m}^{2}\right]$

$L_{T P B} \quad$ Effective TPB interface length per unit volume $\left[\mu \mathrm{m} / \mu \mathrm{m}^{3}\right]$ partial pressure of species $i[\mathrm{~Pa}]$

Gas constant $[\mathrm{J} /(\mathrm{K} \cdot \mathrm{mol})]$

Temperature [K]

Void ratio

Overvoltage of species $i[\mathrm{~V}]$

Potential energy of species $i[\mathrm{~J} / \mathrm{mol}]$

Conductivity of species $i[\mathrm{~S} / \mathrm{m}]$

Tortuosity factor

\section{References}

Chan, H. S., and Z. T. Xia; "AAnode Micro Model of Solid Oxide Fuel Cell," Journal of Electrochemical Society, 148, 388-394 (2001)

Chen, K., X. Chen, Z. Li. N, Ai, X. Huang and W. Su; "Performance of an Anode Supported SOFC with Anode Functional Layers," Electrochimical Acta, 58, 78257830 (2008)

De Boer, B.; "SOFC Anode Hydrogen Oxidation at Porous Nickel and Nickel/Yttria-Stabilised Zirconia Cermet Electrodes" PhD Thesis, University of Twente, the Nether Land (1998)

Konno, A., H. Iwai, M. Saito and H. Yoshida; "Effect of Characteristic Lengths of Electron, Ion and Gas Diffusion on Electrode Performance and Electrochemical Reaction Area," Thermal Science and Engineering, 18, 103-114 (2010)

Mizusaki, J., K. Amano, S. Yamauchi and K. Fueki; "Electrode Reaction at Pt, O2 (g)/Stabilized Zirconia Interfaces. Part I: Theoretical Consideration of Reaction Model," Solid State Ionics, 22, 313-322 (1987)

Murayama, Y., K. Hosoyama, Y. Shimamoto and T. Nakagaki; "Improvement of Power Density and TPB Length by Inserting AFL in SOFC," 2015 SCEJ 47th Annual Meeting, Sapporo, Japan (2015)

Shikazono, N., Y. Sakamoto, Y. Yamaguchi and N. Kasagi; "Effects of Electrode Microstructure on Polarization Characteristics of SOFC Anode," Transaction of JSME(B), 75, 129-136 (2009) 